\section{Verstöße werden abgemahnt}

Mit den Ophthalmologen und den Gynäkologen schneiden in der Beurteilung durch die Patienten nach dem Urteil der VZ NRW zwei Facharztgruppen besonders schlecht ab. Nachweisliche VerstöBe aufgrund von Forumsbeschwerden habe die VZ NRW bereits erfolgreich abgemahnt, wie sie betont. „Nun gilt es, die Patientenrechte zu stärken und die Ergebnisse für eine Verbesserung der Geschäftsbeziehungen von Patienten und Ärzten auf dem Gesundheitsmarkt zu nutzen“, postuliert Schuldzinski - garniert mit dem Hinweis der VZ NRW auf den teilweise umstrittenen medizinischen Nutzen einzelner Angebote. Dies stellt eine Art Selbstreferenz auf den vom Medizinischen Dienst des GKVSpitzenverbandes etablierten - und von dem Verbraucherzentrale Bundesverband unterstützten - „IGeL-Monitor“ dar, bei dem nur wenigen Selbstzahlerangeboten aus wissenschaftlicher Sicht ein Nutzen zuerkannt wird (www.igelmonitor.de). Dies gipfelte vor Kurzem in einer Kontroverse mit der Deutschen Gesellschaft für Hals-Nasen-Ohrenheilkunde, Kopf- und Halschirurgie und dem Deutschen Berufsverband der HNO-Ärzte, die das negative Votum des Portals der Glukokortikoid-Therapie bei Hörsturz kritisierten, da eine neue Leitlinie nicht berücksichtigt worden war. Auch andere Berufsverbände der betroffenen Fachgruppen haben sich zu Wort gemeldet und die pauschale Kritik an der IGeL-Praxis zurückgewiesen.

Matthias Wallenfels

\title{
Angestellte Ärzte bringen keinen Bonus
}

\section{Die Zahl angestellter Ärzte in der ambulanten Versorgung steigt stetig: Waren es 2005 noch rund 8.500, zählte die KBV in 2013 bereits über 22.300 angestellte Mediziner. Die Produktivität der Praxen steigt deshalb aber nicht.}

$\mathrm{D}$ ie wachsende Zahl der angestellten Ärzte bringt den Praxisinhabern laut Studie des Zentralinstituts für die kassenärztliche Versorgung ( $\mathrm{Zi})$ keine nennenswerte Erhöhung ihres Jahresüberschusses. Umsatz und Kosten hielten sich vielmehr die Waage. Bemerkenswert ist dabei, dass in den Praxen mit angestellten Ärzten häufig die Arbeitszeit des Praxisinhabers sogar höher ist als in den Praxen ohne angestellten Kollegen.

Generell gilt, dass die Praxisinhaber im Schnitt auf eine Arbeitszeit von über 50 Wochenstunden kommen, während die angestellten Ärzte oft in einem Umfang von 40- oder 20-Wochenstunden tätig sind. „Die Anstellung von Ärzten führt nicht dazu, dass die Praxisinhaber weniger arbeiten", sagt Zi-Geschäftsführer Dr. Dominik von Stillfried. Die Arbeitszeit der Praxischefs sei nach wie vor der wichtigste Erklärungsfaktor für die Höhe des Praxisumsatzes und damit für die Leistungsfähigkeit der Praxis.

Ein Blick in einzelne Fachgruppen verdeutlicht jedoch, dass die Anstellung doch einen etwas höheren Jahresüberschuss bringt, nicht nur Mehrarbeit. Bei den Hausärzten liegt dieser je Praxisin- haber in Praxen ohne angestellte Ärzte nach der aktuellen Erhebungswelle des Zi-Praxis-Panels bei durchschnittlich $142.500 €$, in Praxen mit angestellten Ärzten bei $178.000 €$. Dabei arbeiten Praxisinhaber mit angestellten Ärzten pro Jahr rund 150 Stunden mehr als Kollegen, die alleine in der Praxis tätig sind. Der Jahresüberschuss je Inhaberstunde liegt bei den Praxisinhabern mit Angestellten bei $69,30 €$, bei den Einzelkämpfern bei 60,09€.

Bei Orthopäden ist der Unterschied sogar noch deutlicher: Der Jahresüberschuss je Inhaberstunde mit angestellten Ärzten liegt bei $112,78 €$, bei den Praxisinhabern, die allein tätig sind, bei 70,62€. Dieser Unterschied hat auch mit dem unterschiedlichen Leistungsspektrum der Praxen zu tun. Auch bei Augenärzten gibt es deutliche Unterschiede.

Rebekka Höhl/Hauke Gerlof

\section{Praxisübernahme nur mit Sprechstundenbedarf}

Wer die Praxis eines Kollegen übernehmen will, sollte auch den Sprechstundenbedarf des Vorgängers im Auge haben, um sich unangenehme Überraschungen zu ersparen. Er habe den Fehler gemacht, sich um das Thema Sprechstundenbedarf nicht zu kümmern, berichtete Dr. Mahmoud Delonge bei der Veranstaltung "Existenzgründung 2.0" der Deutschen Apotheker- und Ärztebank, der ETL Steuerberatungsgesellschaft und der Ärzte Zeitung in Köln. „Ich habe einfach nicht gewusst, dass man am Anfang kei- nen Sprechstundenbedarf bestellen darf", so Delonge.

Bis auf Röntgenkontrastmittel und Impfstoffe müssen Ärzte zu Anfang ihrer vertragsärztlichen Tätigkeit den Sprechstundenbedarf selbst beschaffen. Er gilt als Grundausstattung der Praxis. Die erstmalige Verordnung ist erst zum Ende des ersten Abrechnungsquartals möglich. Die KV Nordrhein weist darauf hin, dass bei einer Praxisübernahme der Vorgänger aber die zuletzt verbrauchten Materialien noch einmal als Sprechstunden- bedarf bestellen kann. „Mein Vorgänger hatte nichts mehr, nicht einmal mehr Kompressen", sagte Delonge. Das war teuer für ihn: Er musste $3.000 €$ selbst bezahlen.

Bei einer Praxisübernahme sei es grundsätzlich wichtig, ein ordentliches Inventarverzeichnis anzulegen, betonte Jens-Peter Jahn von der Anwaltskanzlei Dr. Halbe. "Man muss das Anlagenverzeichnis nehmen, durch die Praxis gehen und sehen, ob die Sachen da sind." Ilse Schlingensiepen 